\title{
Hydrogen Embrittlement and Sub-Critical Crack Growth in Hydride Forming Metals
}

\author{
A.G. Varias \\ Materials Science, Technology and Society, Malmö University, SE 205 06, Malmö, Sweden \\ email: andreas.varias@ts.mah.se
}

\begin{abstract}
Hydride-induced sub-critical crack growth is investigated under conditions of constant temperature, constant crack velocity, plane strain, small-scale hydride precipitation and small-scale yielding. The coupling of the operating physical processes of hydrogen diffusion, hydride precipitation and elastic-plastic material deformation is taken into account.

It is shown that the near-tip field, when quantities are appropriately normalized, depends on a normalized stress intensity factor, which incorporates both effects of the applied stress intensity factor and the crack velocity. When the normalized stress intensity factor tends to zero, the near-tip field of a crack, propagating near the threshold, is derived. Increase of the normalized stress intensity factor produces the evolution of the near-tip field as the velocity of the crack and the applied loading increase, leading to propagation from stageI to stage-II regime. The near-tip field depends strongly on the hydrogen concentration, far from the crack tip.

It is also shown that, as the normalized stress intensity factor tends to zero, a hydrostatic stress plateau develops in the area of hydride precipitation near the tip. The plateau is a result of approaching hydrogen chemical equilibrium in the solid solution. The plateau hydrostatic stress depends strongly on the remote hydrogen concentration. However, it is independent of the plastic properties of the solid solution. Therefore for a given value of the remote hydrogen concentration, the same hydrostatic stress develops near the crack tip either the material is elastic or elastic-plastic as well as for any level of hardening. The same hydrostatic stress develops also behind the crack tip in the presence of hydrides.

As the normalized stress intensity factor increases, hydrogen distribution deviates from that under chemical equilibrium. The hydrostatic stress also deviates from the thermodynamically required constant value and the stress distributions tend to those of a hydrogen free material.

Based on the characteristics of the near-tip field, a critical remote hydrogen concentration has been derived, below which near-tip hydride precipitation does not occur and therefore sub-critical crack growth is suppressed for any level of the applied stress intensity factor. Also fracture criteria are developed, for a remote hydrogen concentration above the critical value, which allow the estimation of the threshold stress intensity factor and the stage-II crack growth velocity.
\end{abstract}


The model has been applied to Zircaloy- 2 and $\delta$-hydride and the predictions on the threshold stress intensity factor and stage-II crack growth velocity are in agreement with experimental measurements.

\section{INTRODUCTION}

Electron microscopy studies have shown that hydrogen embrittlement of hydride forming metals may occur by the precipitation of brittle hydrides at stress concentration locations (e.g. /1-3/). An important feature of hydride-induced embrittlement is the coupling of the operating mechanisms. Indeed, hydrogen diffusion is driven by the gradients of its chemical potential and temperature (e.g. /4/). Note that hydrostatic stress reduces the chemical potential of hydrogen in the solid solution /5/ and creates a hydrogen flux towards stress concentrators. When the terminal solid solubility of hydrogen in the metal is reached at the stress concentration locations, hydrides precipitate. In addition, the terminal solid solubility depends on temperature as well as on stress. The effect of stress on hydrogen terminal solid solubility is caused by the expansion of the hydride phase, during precipitation, as well as by the expansion of the metal lattice, during hydrogen dissolution /6/. Finally, the deformation of the material depends on all other processes due to the change of its volume, which is caused by hydride formation, hydrogen dissolution and temperature variation, as well as on hydride fracture and crack growth.

Varias and Massih /6-8/ developed a model, which takes into account the coupling of all operating physical processes, i.e. hydrogen diffusion, hydride precipitation, non-mechanical energy flow and hydride/solid-solution deformation. Material damage and crack growth initiation were also simulated by using a new version of the de-cohesion model, which takes into account the time variation of energy of decohesion, due to the time-dependent process of hydride precipitation. The bulk of the material, outside the de-cohesion layer, was assumed to behave elastically. The present study extends the Varias and Massih model by considering continuous crack growth in an elastic-plastic material. Emphasis is placed on crack growth under constant or slowly varying velocity, plane strain conditions and constant temperature.

Experiments reveal that hydride-induced sub-critical crack growth is a rather discontinuous process. In some cases, this discontinuous process is obvious, being associated with stepwise decrease of the potential across the mouth of the monitored crack and formation of striations on the fracture surface (e.g. /9/). In other cases, however, of crack growth in metals of relatively large yield stress or under relatively large applied stress intensity factor, the change of the potential is rather continuous and parallel striations are not always obscrved on the fracture surface (e.g. /10/). In the first case, a relatively large and dense hydride cluster, of the size of interstriation spacing, develops ahead of the crack tip; it fractures and the crack is arrested when the ductile metal is reached; the crack grows by repetition of this cycle. In the second case, a relatively small hydride region develops ahead of the crack tip. The crack then propagates by the fracture of this small region and therefore no clear steps in the potential, across the mouth of the crack are detected. Indeed, although there are no clear striations, the fracture surface is made of very small cleavage areas $/ 10 /$. 
In any case, the simulation of hydride-induced steady state crack growth provides important information, such as the extent of the hydride-rich zone near the crack tip and the level of the stresses, which dominate in the hydride precipitation zone. The characteristics of the steady-state field can be used for the prediction of threshold stress intensity factor and stage-II crack growth velocity.

The structure of the paper is as follows. In section 2, the boundary value problem of a steadily propagating crack is described. The governing equations are given in section 3. A discussion on the nondimensional form of the governing equations and the implications on the structure of the near-tip field is included. The near-tip field is discussed in section 4. Analytical expressions of the hydrostatic stress in the hydride precipitation zone around the crack tip are presented, when hydrogen chemical equilibrium is approached, near the propagation threshold. This discussion provides the basis for understanding the strong influence of hydride precipitation on near-tip hydrostatic stress. The implications of the near-tip field on fracture are discussed in section 5, where two fracture criteria are given for growth near the threshold stress intensity factor as well as within stage-II regime. Finally section 6 contains the conclusions.

\section{BOUNDARY VALUE PROBLEM}

A crack is moving with a constant speed $V_{c}$, under plane strain conditions and constant temperature (Figure 1). A Cartesian coordinate system, $\left(x_{1}, x_{2}\right)$, is considered, which has origin at the moving crack tip. $x_{1}$ is the direction of crack propagation. The respective cylindrical coordinates are $(r, \theta)$, where the radius $r$

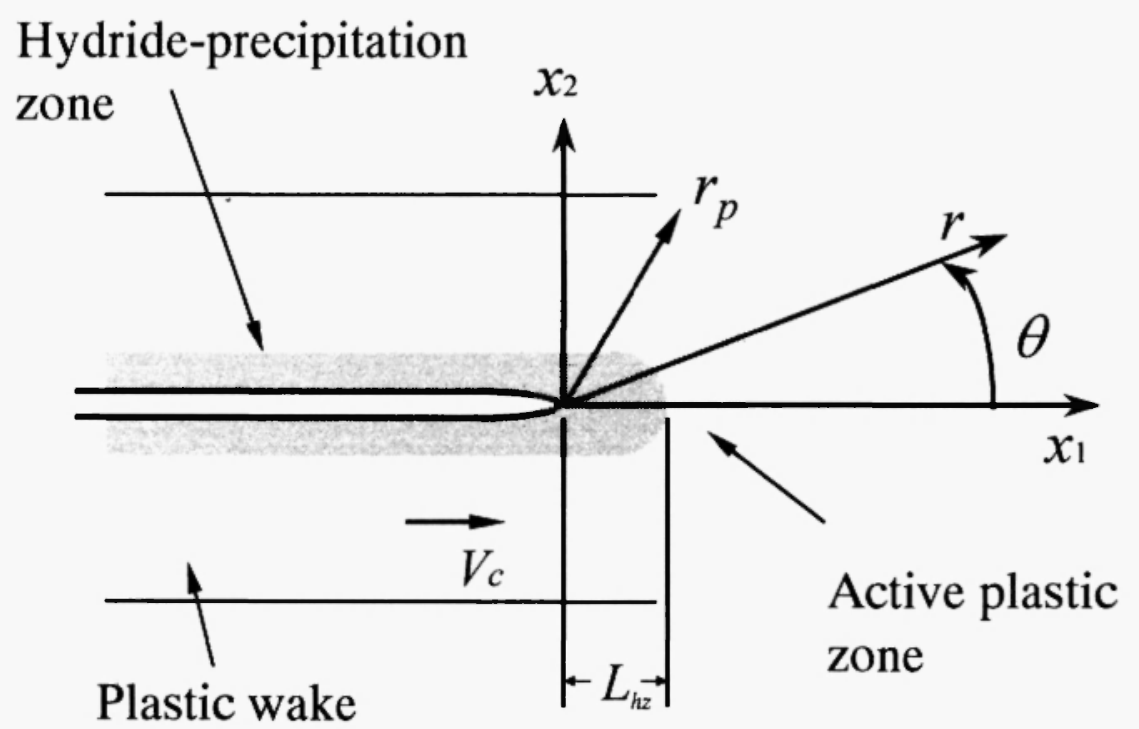

Fig. 1: A crack propagates with a constant velocity, $V_{c}$, in an elastic-plastic material, due to the precipitation and fracture of hydrides, near the tip. $r_{p}$ is the size of the active plastic zone. $L_{h z}$ is extent of the hydride precipitation zone, ahead of the crack tip on the crack plane. 
is measured from the crack tip and the angle $\theta$ is measured from the crack plane. Under steady-state crack growth conditions, considered in the present study, an observer, moving with the crack tip, does not see any change in the distribution of a field quantity. Then, a field quantity, $q$, should only depend on position with respect to the crack tip and not on time, ie. $q=q\left(x_{1}, x_{2}\right)$. Consequently, the material time derivative and the spatial gradient of $q$ satisfy the following well-known relations: $d q / d t=-V_{c}\left(\partial q / \partial x_{1}\right)$ and $\partial q / \partial X_{t}=\partial q / \partial x_{l}$, where $X_{i}$ are the coordinates of a material particle with respect to a system fixed in space. Note that, when the partial time derivative can be neglected with respect to the $x_{1}$-derivative due to the singularity of the crack tip, the steady-state solution is asymptotically valid even under varying crack velocity (e.g. /11/). Therefore, the results, derived for steady-state crack growth conditions, could also be used under slowly varying velocity. In the present analysis, these results are used for deriving information on stage-I crack growth and the threshold stress intensity factor. It is assumed that the crack velocity decreases, thus approaching initially stage-I from stage-II growth and subsequently arrest and thus the threshold stress intensity factor.

Far from the crack tip, at a distance which is large compared to the size of the plastic zone, $r_{p}$, and the hydride-precipitation zone, $L_{h z}, K$-filed dominates and the stress, $\tau_{y}$, is given by the following relation:

$$
\sigma_{i j}=\frac{K_{I}}{\sqrt{2 \pi r}} f_{i j}(\theta), \quad r \gg r_{p}, \quad r \gg L_{h z}
$$

The italic indices of the tensors range from one to three. $K_{I}$ is the mode-I stress intensity factor and $f_{i j}$ is the respective well-known angular stress distribution (e.g. /12/). Also far from the crack tip the concentration of hydrogen, $C^{H}$, is constant and equal to $C_{b}^{H}$ :

$$
C^{H}=C_{b}^{H}, r \gg r_{p}, \quad r \gg L_{h z} .
$$

In the present study, it is assumed that, far from the crack tip, hydrogen is in solid solution and therefore $C_{b}^{H}$ is below the terminal solid solubility of hydrogen in the metal. Between the crack faces it is assumed that the hydrogen gas is in chemical equilibrium with the remote hydrogen concentration. The hydrogen gas is also assumed to be in chemical equilibrium with the hydrogen, which is in solid solution on the fracture surface. Therefore, along the crack faces, the following boundary condition is applied:

$$
C^{H}(r, \pm \pi)=C_{\dot{\Sigma}}^{-} \exp \left[\frac{V^{H}}{3 R T} \sigma_{k k}(r, \pm \pi)\right]
$$

where the repeated italic indices denote summation. $R, \mathrm{~T}$ and $\bar{V}^{i i}$ are the gas constant, the absolute temperature and the molal volume of hydrogen in the solid solution, respectively. It is emphasized that relation (3) does not imply chemical equilibrium of hydrogen in solid solution everywhere in the metal. 
Relation (3) corresponds to the case of a structure in a hydrogen atmosphere. However, hydrogen gas, between the crack faces, is also expected, when hydrogen is already in the metal or is generated by an oxidation process. The effect of hydrogen boundary conditions along the crack faces has been investigated by considering zero hydrogen flux normal to the crack faces $/ 13 /$. It was shown that, for growth near the threshold stress intensity factor, the effect of crack face boundary conditions on near-tip field is negligible. Consequently the estimate of the threshold stress intensity factor, based on the calculated near-tip field, does not depend on crack face boundary conditions. For growth, away from the threshold, the stress and hydride volume fraction distributions are only weakly affected. However, there are no implications on the prediction of stage-II crack growth velocity $/ 13 /$.

Due to symmetry with respect to the crack plane, the shear stress, as well as the displacement and the flux of hydrogen, normal to the crack plane, are zero along the crack line.

\section{GOVERNING EQUATIONS}

The governing equations of hydrogen diffusion, hydride precipitation and material deformation are discussed in the following sections. The model is developed for a metal, $\mathrm{M}$, which forms hydrides of the type $\mathrm{MH}_{\mathrm{x}} ; \mathrm{x}$ is the mole fraction of hydrogen in the hydride. The presence of the hydrides is described by the hydride volume fraction; the hydrides are smeared. The development of regions rich in hydrides, their shape and size is given by the distribution of hydride volume fraction.

The finite element implementation of the governing equations is discussed in $/ 13 /$.

\subsection{Hydrogen Diffusion and Hydride Precipitation}

The governing equation of hydrogen diffusion is the differential form of hydrogen mass conservation. Taking into account steady-state crack growth, one derives:

$$
V_{c} \frac{\partial C^{H T}}{\partial x_{1}}=\frac{\partial J_{k}^{H}}{\partial x_{k}}
$$

The total hydrogen concentration, $C^{H T}$, is related to the concentration of hydrogen in the solid solution, $C^{H}$, and the hydride, $C^{H, h r}$, as follows:

$$
C^{I T T}=f C^{H \cdot h r}+(1-f) C^{H}
$$

where $f$ is the volume faction of the hydrides. $C^{H}$ is defined with respect to the volume occupied by the solid solution, i.e. $(1-f) V$. Then, $C^{H}$ is equal to the hydrogen terminal solid solubility, $C^{T S}$, when $f \neq 0$. 
Similarly $C^{I I, h i r}$ is defined with respect to the volume occupied by the hydrides, i.e. $f V$, and therefore it can be considered constant.

Hydrogen diffusion in the hydride is very slow, when compared to the diffusion in the solid solution, and therefore neglected in the present analysis. Then, hydrogen flux is given by the following relation:

$$
J_{k}^{H}=-(1-f) \frac{D^{H} C^{H}}{R \mathrm{~T}} \frac{\partial \mu^{H}}{\partial x_{k}},
$$

where $D^{H}$ and $\mu^{H}$ are the diffusion coefficient and the chemical potential of hydrogen in the solid solution. The effect of stress on hydrogen diffusion is introduced by hydrogen chemical potential /5/:

$$
\mu^{H}=\mu^{H, 0}-\frac{\sigma_{k k}}{3} V^{H},
$$

where $\mu^{H, 0}$ is the chemical potential of hydrogen in the solid solution, under stress-free conditions, for the same concentration as that under stress. It is assumed that $\mu^{\prime \prime, 0}$ depends on hydrogen concentration, according to the law of ideal solutions. In relation (7) higher order stress terms have been neglected $/ 6 /$. Also it is assumed that hydrogen concentration has no effect on solid solution elastic stiffness.

In the present analysis, hydride/solid-solution phase transformation is assumed to be a reversible process. Therefore the precipitation of a small hydride, which corresponds to a negligible increase of the hydride volume fraction, is accommodated nearly elastically. However, the growth of the hydrides eventually leads to irreversible metal plastic deformation, depending on hydride expansion during precipitation. This effect is taken into account by the governing equations of material deformation, described in section 3.2. Under these assumptions, the terminal solid solubility of hydrogen in the stressed metal is given, by the following relation:

$$
\begin{aligned}
& C^{T S}=C_{z}^{T S} \exp \left(\frac{\bar{v}_{\mathrm{int}}}{\mathrm{x} R \mathrm{~T}}\right) \exp \left(\frac{\sigma_{m m} \bar{V}^{H}}{3 R \mathrm{~T}}\right),
\end{aligned}
$$

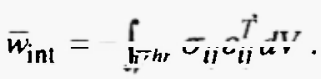

In (8a), higher order stress terms have been neglected; the interested reader is referred to the discussion and derivation in $/ 6 / w_{\text {int }}$ is the interaction strain energy per mole of hydride, of volume $\bar{V}^{h r}$. Note that hydride formation is accompanied by a deformation, $\varepsilon_{l j}^{T}$, which is mainly a volume expansion. $\sigma_{i j}$ is the applied stress field. Finally, $C_{e}^{T S}$ is the terminal solid solubility under no external load and includes the work of hydride accommodation. The first exponential term in (8a) is due to the reduction of the chemical potential of 
the hydride phase by the externally applied tensile stress field. On the other hand, the second exponential term in (8a) is due to the reduction of the chemical potential of the hydrogen in solid solution by the externally applied tensile stress field. These two events have opposite influence on the phase transformation and consequently on hydrogen terminal solid solubility. Indeed, when the externally applied hydrostatic stress increases, the terminal solid solubility decreases due to the first exponential term, favoring hydride formation, while it increases due to the second exponential term, favoring hydrogen in solid solution. In Zircaloy-2 with the properties given in Table 1, the interaction strain energy term is dominant.

Note that the present model predicts hydride stability with material plastic deformation. Indeed, according to the results, which are discussed in following sections, hydrides exist along the crack faces, in the case of an elastic-plastic material, depending on hardening and remote hydrogen concentration, while the hydrides re-dissolve completely, after fracture, in the case of a purely-elastic material, in agreement with experimental observations (e.g. /1/,/10/).

\subsection{Material Deformation}

The material, composed of solid solution and hydrides, is assumed to be elastic-plastic. The deformation is coupled with hydrogen diffusion and hydride precipitation due to the strains, which are caused by hydride formation and hydrogen dissolution:

$$
\begin{aligned}
& \sigma_{i j}=D_{i j k l}^{c}\left(\varepsilon_{k l}-\varepsilon_{k l}^{\mu}-\varepsilon_{k l}^{i \ddot{ }}\right), \\
& D_{i j k l}^{p}=\lambda \delta_{i j} \delta_{k l}+\mu\left(\delta_{l k} \delta_{j l}+\delta_{i l} \delta_{j k}\right), \\
& \varepsilon_{k l}^{H}=\frac{1}{3} \delta_{k l}\left[f \theta^{h r}+(1-f) C^{H} V^{H}\right] .
\end{aligned}
$$

$D_{i j k l}^{e}$ is the elastic stiffness; $\lambda$ and $\mu$ are Lame constants, of the solid-solution/hydride composite. $\varepsilon_{k l}, \varepsilon_{k l}^{p}$ and $\varepsilon_{k l}^{H}$ are the total strain, the plastic strain and the strain caused by hydride formation and hydrogen dissolution, respectively. Note that $\theta^{h r}\left(=\varepsilon_{k k}^{T}\right)$ is the hydride expansion strain.

The effect that hydrogen concentration, mainly hydride volume fraction, may have on elastic-plastic material properties is not taken into account in the present analysis. The numerical simulations have been performed, by assuming that the elastic-plastic properties of the solid-solution/hydride composite are identical to those of the metal. The effect of hydride volume fraction on the deformation properties of the composite and the implications on the near-tip field is discussed in $/ 14 /$, where different hydride and solid solution elastic properties are considered and a composite material model is employed. According to the discussion in $/ 14$ / as well as in section 4.1 of the present work, the near-tip constant hydrostatic stress, which 
develops as the sub-critical crack growth threshold is approached, depends only weakly on the elastic-plastic properties of the hydrides and the solid solution.

The plastic deformation of the material is governed by $J_{2}$-flow theory:

$$
d \varepsilon_{i j}^{p}=\frac{3 \sigma_{i i}^{\prime} d \sigma}{2 \bar{\sigma}\left(d H / d \bar{\varepsilon}^{p}\right)}
$$

where $\sigma_{i j}^{\prime}\left(=\sigma_{i j}-(1 / 3) \sigma_{k k} \delta_{i j}\right)$ is the stress deviator, $\sigma\left(=\sqrt{3 / 2}\left(\sigma_{i j}^{\prime} \sigma_{i j}^{\prime}\right)^{1 / 2}\right)$ is the effective stress and $\bar{\varepsilon}^{p}$ is the accumulated effective plastic strain $\left(d \varepsilon^{p}=\sqrt{2 / 3}\left(d \varepsilon_{i j}^{p} d \varepsilon_{i j}^{p}\right)^{1 / 2}\right)$. Isotropic material hardening is assumed according to the relation, $\sigma=H\left(\bar{\varepsilon}^{p}\right)$. In the present model, the power-law hardening relation, used in previous steady-state crack growth studies is considered /15-16/:

$$
\left(\frac{\vec{v}}{\sigma_{0}}\right)^{1 / N}-\frac{\bar{\sigma}}{\sigma_{0}}=\frac{3 E}{2(1+v)} \frac{\bar{\varepsilon}^{p}}{\sigma_{0}}, \quad \bar{\sigma} \geq \sigma_{0}
$$

$E, v$ are Young's modulus and Poisson's ratio of the material, related to $\lambda$ and $\mu$ by the well-known elasticity relations. $N$ is the hardening exponent, which takes values in the range $(0,1) . N=1$ corresponds to an elastic material and $N=0$ corresponds to a perfectly plastic material. Finally $\sigma_{0}$ is the yield stress in tension.

\subsection{Non-Dimensional Forms of Governing Equations - Implications on Steady-State Crack Growth Solutions}

The size of the area over which hydrogen redistributes during crack propagation depends on the ratio of the hydrogen diffusion coefficient and the resulting crack tip speed. Therefore $D^{H} / V_{c}$ is a characteristic length of hydride induced sub-critical crack growth. Also, a characteristic level of hydrogen concentration is the terminal solid solubility under no applied stress, $C_{e}^{T S}$. Finally, $R \mathrm{~T} / \bar{V}^{i i}$ is a characteristic stress, introduced by hydrogen flux. Note that, according to the discussion in section $4.1, R \mathrm{~T} / V^{H}$ is proportional to the hydrostatic stress in the zone of hydride precipitation, under hydrogen chemical equilibrium. Then, by using the following normalization:

$$
\tilde{x}_{i}=x_{i} \frac{V_{i}}{\bar{\nu}^{\underline{H}}}
$$




$$
\begin{aligned}
& \bar{C}^{H}=\frac{C^{H}}{C^{T S}}, \\
& \ddot{\sigma}_{i j}=\sigma_{i j} \frac{\bar{V}^{H}}{R} \overline{\mathrm{T}},
\end{aligned}
$$

one derives the governing equations of hydrogen diffusion and hydride precipitation, based on dimensionless quantities:

$$
\begin{aligned}
& \frac{\partial \tilde{C}^{H T}}{\partial \tilde{x}_{1}}=\frac{\partial \tilde{J}_{k}^{H}}{\partial \tilde{x}_{k}}, \\
& \tilde{J}_{k}^{H}=\frac{J_{k}^{H}}{C_{e}^{T \tilde{V}_{\tilde{v}_{c}}}}=-(1-f)\left(\frac{\partial \tilde{C}^{H}}{\partial \tilde{x}_{k}}-\frac{\tilde{C}^{H}}{3} \frac{\partial \tilde{\sigma}_{m m}}{\partial \tilde{x}_{k}}\right), \\
& \tilde{C}^{H T}=\frac{C^{H T}}{C_{z}^{T S}}-f \frac{\mathrm{x}}{C_{2}^{T S} \bar{V}^{h r}}+(1-f) \tilde{C}^{\ddot{i}}, \\
& \tilde{C}^{T S}=\frac{C^{T S}}{C_{e}^{T S}}=\exp \left[\frac{\tilde{\sigma}_{m m}}{3}\left(1-\frac{\theta^{h r} \bar{V}^{h r}}{\mathrm{x} \bar{V}^{H}}\right)\right] .
\end{aligned}
$$

Relations (9a-b), (10) and (11), which govern deformation, do not change from when normalization (12) is used. Certainly, Young's modulus and the yield stress in tension have to be normalized, according to relation (12c). Note also that hydrogen-induced expansion takes the following form:

$$
\varepsilon_{k l}^{H}=\frac{1}{3} \delta_{k l}\left[f \theta^{h r}+(1-f) \tilde{C}^{H} C_{e}^{T S} \bar{V}^{H}\right]
$$

One observes that the coefficient of hydrogen diffusion and the velocity of the crack are not present in the non-dimensional forms of the governing equations. Therefore the effect of hydrogen diffusion coefficient and crack tip velocity is implicit. This effect can be derived through the dependence of the normalized quantities on $D^{H}$ and $V_{c}$.

Based on (13) and (14) one concludes that the near tip field, ahead of a steadily propagating crack, has the following form:

$$
\tilde{u}_{t}=U_{\imath}\left(\tilde{x}_{k} ; \tilde{K}_{I}, \tilde{C}_{b}^{H}\right)
$$




$$
\begin{aligned}
& \bar{\sigma}_{i j}=S_{i j}\left(\tilde{x}_{k} ; \tilde{K}_{I}, \tilde{C}_{b}^{H}\right), \\
& \bar{C}^{H}=C\left(\bar{x}_{k} ; \tilde{K}_{I}, \dot{C}_{b}^{H}\right), \\
& f=F\left(\bar{x}_{k} ; \tilde{K}_{I}, \tilde{C}_{b}^{H}\right),
\end{aligned}
$$

where displacements have been normalized in the same way as particle coordinates, $\tilde{u}_{i}=u_{i} \cdot\left(V_{c} / D^{H}\right)$. The near-tip distributions of the normalized quantities depend on the normalized boundary conditions: $\tilde{K}_{I}=K_{I} \sqrt{V_{c} / D^{H}} /\left(R \mathrm{~T} / \bar{V}^{H}\right)$ and $\tilde{C}_{j}^{H}=C_{j}^{H} / C_{e}^{T S}$. They also depend on the following material parameters: $\tilde{E}, \nu, \bar{\sigma}_{0}, N, \mathrm{x}, \theta^{h r}, C_{e}^{T S} \bar{V}^{H}$ and $C_{e}^{T S} \bar{V}^{h r}$, where $\bar{E}$ and $\tilde{\sigma}_{0}$ are the normalized values of Young's modulus and the yield stress in tension.

It is also interesting to mention that temperature is not present in the non-dimensional forms of the governing equations. Therefore, the effect of temperature on the near-tip distribution of a field quantity is implicit, i.e. only through the above mentioned material parameters, the non-dimensional boundary conditions and relations (12).

Let us consider a crack propagating in a metal with a certain average hydrogen concentration (i.e. certain $C_{b}^{H}$-level), under constant temperature. According to relations (15), an increase of the applied stress intensity factor, $K_{l}$, at a given speed, $V_{c}$ or an increase of the crack tip speed, at a given applied stress intensity factor, lead to the same changes of the near-tip field distributions, when the variation of the normalized stress intensity factor, $\tilde{K}_{I}$, is the same. Note that the crack-tip speed remains nearly constant, while the applied stress intensity factor changes, during stage II sub-critical crack growth. Also the applied stress intensity factor remains nearly the same, while the crack tip speed changes, when the threshold of subcritical crack growth is approached. Therefore, the study of relations (15) provides information on the changes of the near-tip field near the threshold as well as within stage II growth. Actually, the variation of the normalized stress intensity factor, from zero to sufficiently large positive values, produces the evolution of the near-tip field as the velocity of the crack and the applied stress intensity factor increase, leading to propagation from stage-I to stage-II regime. This is the subject of section 4.

\section{NEAR-TIP FIELD DURING HYDRIDE-INDUCED STEADY-STATE CRACK PROPAGATION}

The discussion on the hydrostatic stress level, which develops in the hydride precipitation zone near the crack tip under hydrogen chemical equilibrium, presented in section 4.1, provides a general analysis.

The numerical simulations have been performed for irradiated and therefore of large yield stress Zircaloy- 
2 and $\delta$-hydride $\left(\mathrm{ZrH}_{1.66}\right)$ at $300^{\circ} \mathrm{C}$. The material properties are given in Table 1. Calculations for crack propagation in un-irradiated zirconium alloys with relatively low yield stress at $200^{\circ}$ and $300^{\circ} \mathrm{C}$ are presented in $/ 14 /$.

\section{Table 1}

Material properties used in the finite element calculations. The material properties correspond to irradiated Zircaloy- 2 and $\delta$-hydride $\left(\mathrm{ZrH}_{1.66}\right)$.

\begin{tabular}{ll}
$E, v$ & $80.4 \mathrm{GPa}, 0.369(573 \mathrm{~K})$ \\
$\sigma_{0}$ & $580 \mathrm{MPa}(573 \mathrm{~K})$ \\
$D^{H}$ & $2.17 \cdot 10^{-7} \exp (-35087.06 / R \mathrm{~T}) \mathrm{m}^{2} / \mathrm{s}$ \\
$C_{e}^{T S}$ & $6.3741 \cdot 10^{5} \exp (-34542.75 / R \mathrm{~T}) \mathrm{mole} / \mathrm{m}^{3}$ \\
$C^{H, h r}$ & $1.02 \cdot 10^{5} \mathrm{~mole} / \mathrm{m}^{3}$ \\
$\bar{V}^{H}$ & $7 \cdot 10^{-7} \mathrm{~m}^{3} / \mathrm{mole}$ \\
$V^{h r}$ & $16.3 \cdot 10^{-6} \mathrm{~m}^{3} / \mathrm{mole}$ \\
$\theta^{h r}$ & 0.1636 \\
$\mathrm{X}$ & 1.66 \\
\hline
\end{tabular}

The present results correspond to remote hydrogen concentration, $\tilde{C}_{b}^{\prime \prime}$, equal to 0.94 , which, according to relation (18b), is associated with a stress trace in the hydride precipitation zone, equal to the yield stress in tension. Additional calculations for other values of $\bar{C}_{b}^{i}$ are discussed in /13/. However, the strong effect of the remote hydrogen concentration on the near-tip stress field is revealed in the analysis of section 4.1.

The combined effect of the intensity of the remote $K$-field and the crack tip velocity, on the near-tip field, has been investigated by considering a wide range of $\ddot{K}_{l},(0.0013-0.1334)$. The selected values of $K_{I}$ provide crack tip velocities between $10^{-10}$ and $10^{-6} \mathrm{~m} / \mathrm{s}$, when the stress intensity factor is about $10 \mathrm{MPa} \mathrm{V}_{\mathrm{m}}$, i.e. of the order of the threshold stress intensity factor of zirconium alloys.

\subsection{Hydrostatic Stress Level in the Hydride Precipitation Zone, under Hydrogen Chemical Equilibrium - Implications on Sub-Critical Crack Growth}

Consider a stationary crack, under small scale yielding conditions. Initially, hydrogen redistributes under the influence of the crack-tip field. After sufficient time, chemical equilibrium is reached, hydrogen chemical potential is constant everywhere in the body and there is no further diffusion. Then, in the solid solution, hydrogen concentration is given by the following relation: 


$$
C^{H}=C_{R}^{H} \exp \left[\frac{\bar{V}^{H}}{3 R \Gamma}\left(\sigma_{k k}-\sigma_{k k}^{R}\right)\right]
$$

where $C_{R}^{H}$ and $\sigma_{k k}^{R}$ is the concentration of hydrogen and the stress trace of a reference particle. Hydrogen chemical equilibrium is also valid far from the crack tip, where $K$-field dominates, i.e. for $r \gg r_{p}$ and $r \gg L_{h z}$. Then a particle far from the crack tip could be chosen as a reference particle, in which case, $C_{R}^{H}=C_{b}^{H}$. Note that the stress trace of a particle far from the crack tip is very small, compared to stress trace near the crack tip. Therefore the reference-stress term, in (16), can be neglected, under small scale yielding and $C_{b}^{H I}<C_{e}^{T S}$, when $C_{R}^{H}$ is taken to be equal to $C_{b}^{H}$.

When hydrogen chemical equilibrium is reached in the hydride precipitation zone near the crack tip, the following equality is satisfied in the area, where the volume is only partly occupied by the hydrides:

$$
C^{H}=\mathcal{C}_{\underline{P}}^{H} \exp \left[\frac{\bar{V}^{H}}{3 R \mathrm{~T}}\left(\sigma_{k k}-\sigma_{k k}^{R}\right)\right]=C_{s}^{T S} \exp \left(\frac{\bar{m}_{\text {int }}}{\mathrm{x} R \mathrm{~T}}\right) \exp \left(\frac{\sigma_{k k} \bar{V}^{H}}{3 R \mathrm{~T}}\right) .
$$

After some algebraic manipulations one derives a relation for the stress trace in the zone of hydride precipitation, $\sigma_{k \bar{k}}^{h z}$ :

$$
\sigma_{k k}^{h z}=\left(\frac{3 \mathrm{x}}{\theta^{h r} V^{h r}}\right)\left[\frac{\sigma_{a . m .}^{R} \bar{V}^{H}}{3}+R \mathrm{~T} \ln \left(\frac{C_{o}^{T S}}{C_{R}^{H}}\right)\right],
$$

or :

$$
\sigma_{k k}^{h z} \cong\left(\frac{3 \mathrm{x}}{\theta^{h r} \bar{V}^{h r}}\right) R \mathrm{~T} \ln \left(\frac{C_{e}^{T S}}{C_{b}^{H}}\right)
$$

In (18b), the reference-stress term has been neglected for being very small compared to the contribution of $C_{z}^{T S} / C_{b}^{H}$. Note that the stress trace in the area of hydride precipitation, $\sigma_{k k}^{h z}$, is constant, when hydrogen chemical equilibrium is reached. The level of $\sigma_{k k}^{h z}$ mainly depends on the ratio $C_{e}^{T S} / C_{b}^{H}$ (i.e. on remote hydrogen concentration) as well as on temperature. Because of the linear relation between $\sigma_{k k}^{h z}$ and $R T, \sigma_{k k}^{h z}$ is equivalent to $R \mathrm{~T} / V^{H}$ for normalizing stresses. It is also emphasized that, according to (18b), $\sigma_{k k}^{h z}$ does not depend on the plastic properties of the metal and the hydrides. Extension of the analysis for hydrides of different elastic properties than those of the solid solution shows that the constant stress trace level in the hydride precipitation zone is only weakly dependent on the elastic stiffness of the hydride and the metal /14/. Therefore (18) is valid and provides the same stress trace level, near the crack tip, in a metal, for the whole range of hardening exponents $(1>N>0)$, i.e. from purely elastic to perfectly plastic material behavior. Consequently the dominance of the asymptotic fields of hydrogen-free elastic or elastic-plastic materials is 
lost in the hydride precipitation zone, under hydrogen chemical equilibrium. Relation (18) takes the following form, when the field quantities are normalized according to (12):

$$
\tilde{\sigma}_{k k}^{h z}=\left(\frac{\mathrm{x} V^{H}}{\theta^{h r} V^{h r}}\right)\left[\tilde{\sigma}_{m m}^{R}-3 \ln \left(\tilde{C}_{R}^{H}\right)\right] \cong-\left(\frac{3 \mathrm{x} V^{H}}{\theta^{h r} V^{h r}}\right) \ln \left(\tilde{C}_{3}^{H}\right) .
$$
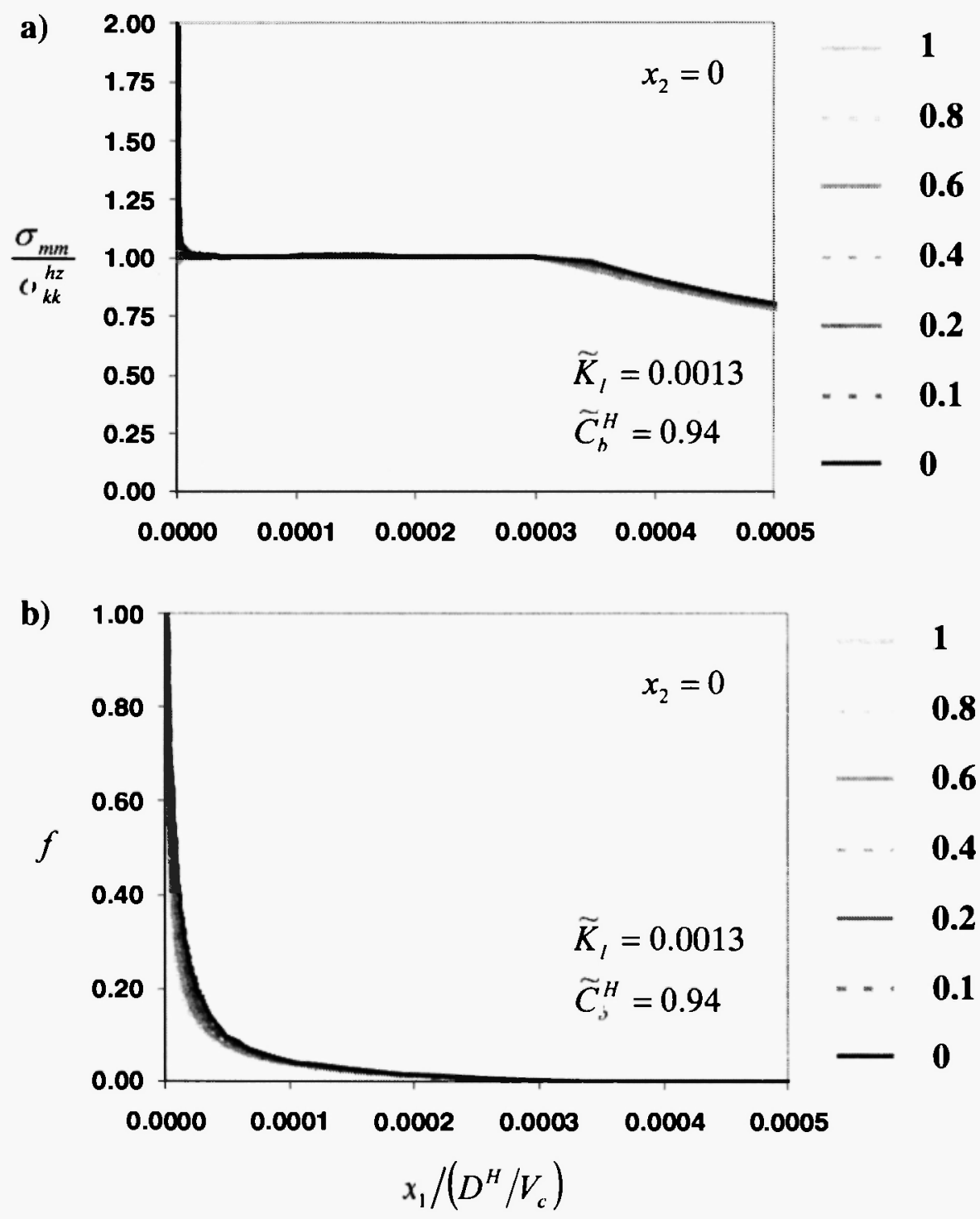

Fig. 2: Normalized distributions of (a) stress trace and (b) hydride volume fraction, ahead of a steadily propagating crack, on the crack plane, in a zirconium alloy near hydrogen chemical equilibrium. $\mathrm{T}=573 \mathrm{~K} . \sigma_{k k}^{h z}$ is calculated by (18b). 
In the case of a steadily propagating crack, one shows, by using the conservation of hydrogen mass, that hydrogen chemical equilibrium and constant hydrostatic stress in the area of hydride precipitation is not strictly satisfied. However, hydrogen chemical equilibrium and consequently constant hydrostatic stress can be approached if $\bar{K}_{l}$ is sufficiently small, i.e. if the crack tip speed, $V_{c}$, is sufficiently small and thus adequate time is provided for the redistribution of hydrogen. Then the crack-tip field for growth near the threshold stress intensity factor is approached. Indeed, the numerical calculations confirm the validity of relations (18) and (19). Figure 2a shows the distribution of stress trace ahead of a crack tip along the crack plane, for steady crack propagation in a zirconium alloy at $300{ }^{\circ} \mathrm{C}$; the numerical stress values have been normalized by the stress trace derived from relation (18b). In this particular example, $\ddot{K}_{I}=0.0013$. Note that the level of the plateau stress is in excellent agreement with relation (18b) and it does not depend on material hardening. The plateau corresponds to the area of hydride precipitation; see Figure $2 b$, where the hydride volume fraction distribution is presented along the crack plane. In an elastic material, the deviation between the numerical values of the stress trace and relation $(18 \mathrm{~b})$ is less than $0.6 \%$ for $x_{1} /\left(D^{H} / V_{c}\right)>4 \cdot 10^{-6}$. In a perfectly-plastic material, the deviation is less than $6 \%$ for $x_{1} /\left(D^{H} / V_{c}\right)>4 \cdot 10^{-6}$ and less than $1 \%$ for $x_{1} /\left(D^{H} / V_{c}\right)>2.6 \cdot 10^{-5}$. Very close to the crack tip, the hydride volume fraction increases significantly, the stress distribution approaches that of a homogeneous material and the stress trace deviates from that given by relation (18b); in this area, due to the steep variation of stress, hydrogen concentration deviates from that expected under chemical equilibrium. Note that, outside the area of steep stress variation, the stress plateau develops everywhere in the hydride precipitation zone and not only ahead of the crack tip. Consequently, even behind the propagating crack, the stress trace is equal to $\sigma_{k k}^{h z}$ in the presence of hydrides. Indeed, according to Figure $3 \mathrm{a}$, the stress trace is constant around the crack tip for any value of hardening exponent from 0 to 0.6 . In the case of $N$ equal to 0.8 and 1 , the stress trace decreases near the crack faces, due to the redissolution of the hydrides (see Figure $3 \mathrm{~b}$ ). Certainly, without the presence of hydrides, the stress trace can vary. In order to appreciate the strong effect of hydride formation on near-tip stress level, Figure 3a also shows the angular distribution of the stress trace, around a propagating crack in an elastic perfectly-plastic hydrogen-free material $/ 17 /$.

Relation (18b) provides information on the size of the area of hydride precipitation ahead of the crack tip. Let us assume clastic material deformation. Hydride precipitation, along the crack plane, approximately starts, when the plateau stress, $\sigma_{k k}^{h z}$, is equal to the stress trace, given by the $K$-field. Then:

$$
L_{n z} \simeq \frac{2}{\pi}(1+\cdots)^{2}\left(\frac{K_{I}}{\sigma_{k k}^{h z}}\right)^{2} .
$$

According to the above relation, the size of the area of hydride precipitation depends on the applied stress intensity factor as well as on the level of stress trace, which is required by hydrogen chemical equilibrium. 
For the zirconium alloy, under consideration, $L_{h z} /\left(K_{I} / \sigma_{k k}^{h z}\right)^{2} \equiv 1.19$. Note that the numerical calculations provide a value of $L_{h z} /\left(K_{I} / \sigma_{k k}^{h:}\right)^{2}$ equal to 1.21 for both elastic and elastic-plastic materials.
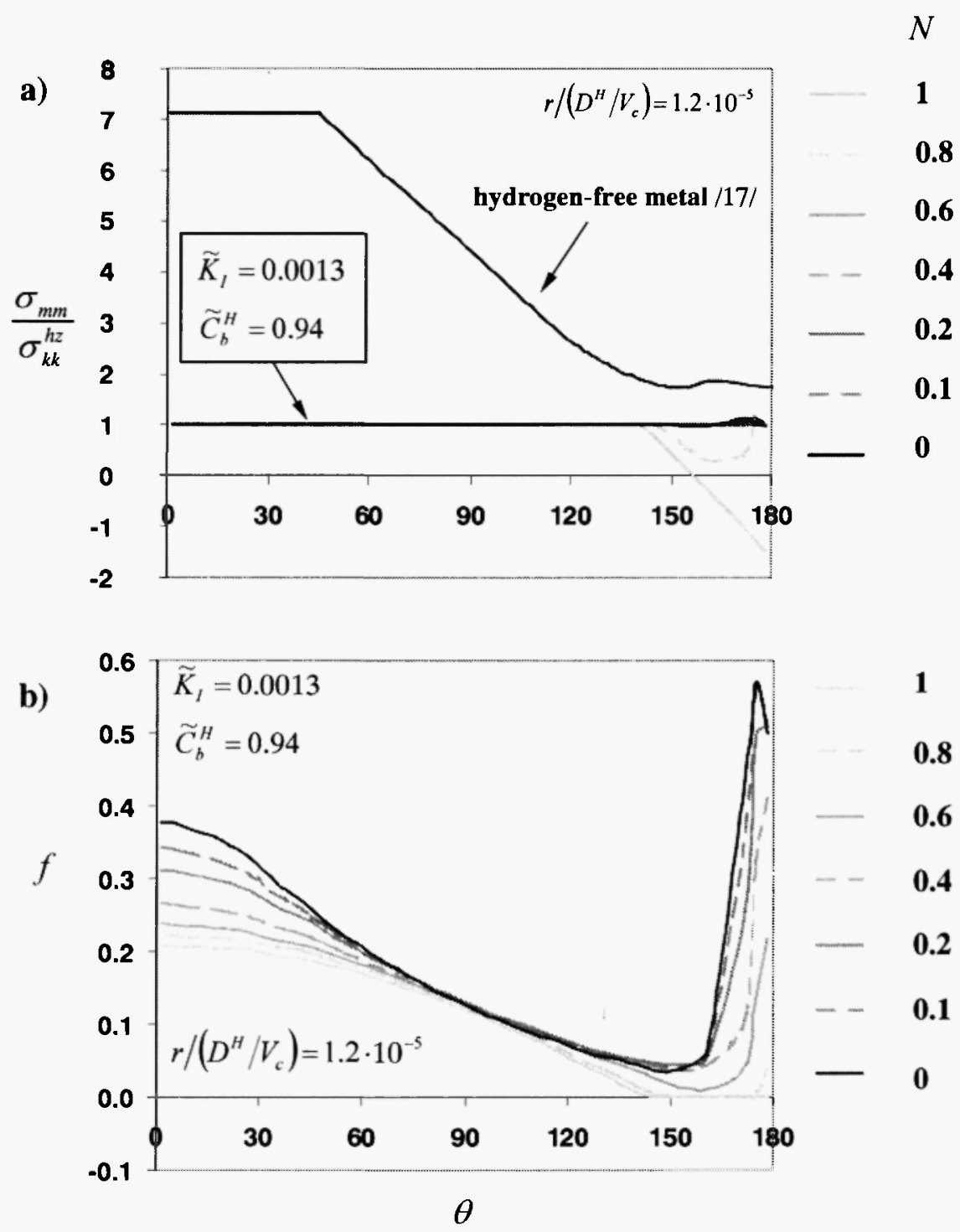

Fig. 3: Angular near-tip distributions of (a) stress trace and (b) hydride volume fraction, in a zirconium alloy. $\mathrm{T}=573 \mathrm{~K} . \sigma_{k k}^{h z}$ is calculated by $(18 \mathrm{~b})$.

Relations (18) and (19) provide also information on the precipitation or not of hydrides in a perfectly plastic material. Indeed, according to the near-tip field of a stationary crack or a crack propagating quasistatically in a non-hardening material, the stress trace level on the crack plane, ahead of the tip, is constant, 
$\sigma_{k k}^{e p} \equiv 7 \sigma_{0}$ (e.g. $\left./ 12 /, / 17 /\right)$. Hydride expansion, during precipitation, is expected to lead to reduction of the stress trace. Therefore, hydride precipitation, under chemical equilibrium, is only possible if the stress trace level, required by hydrogen chemical equilibrium, is smaller than that expected in a hydrogen free material:

$$
\sigma_{k k}^{h \tau}<\sigma_{m m}^{e p}
$$

Satisfaction of relation (21) does not necessarily mean that hydride-induced sub-critical crack growth will occur, because in addition to hydride precipitation, the hydride-rich area should be sufficiently large. On the other hand, if inequality (21) is not satisfied, hydrides do not precipitate ahead of a crack tip in a perfectly plastic material and therefore sub-critical crack growth is suppressed. Then, from (21) one concludes that hydride-induced sub-critical crack growth, under small scale yielding, is suppressed, when the remote hydrogen concentration in a non-hardening metal satisfies the inequality:

$$
\bar{C}_{5}^{H}<\exp \left(-\frac{\tilde{\sigma}_{k k}^{e p} \epsilon^{h r} \bar{\eta}^{h r}}{3 \times V^{H}}\right) .
$$

If the metal hardens, the near-tip hydrogen-free stress trace, $\tau_{\underline{k t}}^{e p}$, is larger than $7 \sigma_{0}$ and consequently the critical hydrogen concentration decreases.

\subsection{Combined Effect of Stress Intensity Factor and Crack Velocity on Near Tip Field}

With the increase of the normalized stress intensity factor, i.e. for crack propagation away from the threshold, the stress trace increases, deviating from the level, which is required by hydrogen chemical equilibrium. Figure 4 shows the distributions of the stress trace, ahead of the crack tip and along the crack

plane for $\mathrm{T}=573 \mathrm{~K}$ and $\tilde{C}_{j}^{H}=0.94$; the distributions for $\tilde{K}_{I}$ equal to $0.0042,0.0133,0.0422$ and 0.0943 are presented. The stress has been normalized by the value of $\sigma_{k k}^{h z}$, which is expected under hydrogen chemical equilibrium (relation (18b)). According to Figure $4 a$, the stress trace is nearly constant in the hydride precipitation zone, when $\bar{K}_{I}=0.0042$, and the effect of material hardening is negligible. The stress level is slightly larger than that required by hydrogen chemical equilibrium; the deviation from $\sigma_{k k}^{h-}$ is about $12 \%$ for a non-hardening material and reduces slightly with the increase of hardening. The plateau stress is lost for larger $\tilde{K}_{l}$-values. However, according to Figures $4 \mathrm{~b}$ and $\mathrm{c}$, the effect of material hardening is still weak for $\bar{K}_{I}$ smaller or equal to 0.0422 . Hardening becomes important for larger values of $\bar{K}_{I}$ (see Figure $4 \mathrm{~d}$ ). Note also that as $\bar{K}_{I}$ increases, the stress trace increases, approaching the levels, which are expected in a hydrogen-free material. In the case of non-hardening behavior, the stress trace tends to $7 \sigma_{0}$. Indeed, the maximum stress trace value, for $N=0$, is equal to $6.81 \sigma_{0}$ and $6.94 \sigma_{0}$, when $\bar{K}_{I}=0.0943$ and 0.1334 , respectively. The stress trace distributions, for relatively large values of $\bar{K}_{I}$ (i.e. $\bar{K}_{I}>0.0943$ ) and non- 
hardening behavior, are also rather flat near the crack tip, i.e. $0.01<x_{1} /\left(D^{H} / V_{c}\right)<0.03$. Thus, not only the maximum value but also a part of the distribution, near the crack tip, approaches the constant stress trace of a hydrogen-free non-hardening material.
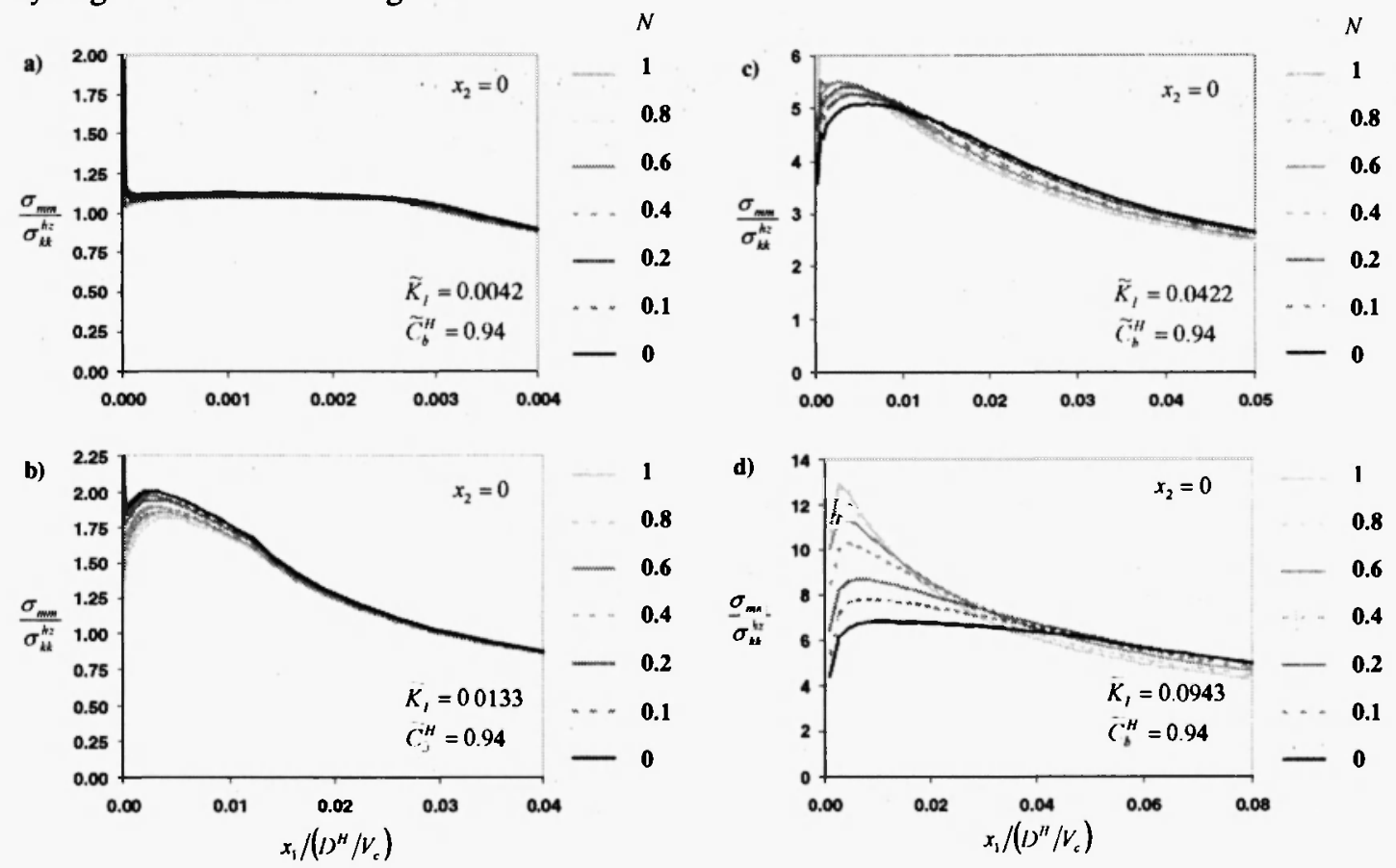

Fig. 4: Normalized distributions of stress trace ahead of a steadily propagating crack, on the crack plane, in a zirconium alloy. $\mathrm{T}=573 \mathrm{~K}$. (a) $\bar{K}_{l}=0.0042$. (b) $\bar{K}_{l}=0.0133$. (c) $\bar{K}_{l}=0.0422$. $\bar{K}_{l}=0.0943 . \sigma_{k k}^{h z}$ is calculated by $(18 \mathrm{~b})$.

According to the analysis of section 4.1, the stress plateau in the hydride precipitation zone, when hydrogen chemical equilibrium is approached, develops in the case of stress trace. There is no requirement of constant values for the stress components. Figure 5 shows the distribution of $\sigma_{11}$ and $\sigma_{22}$ stresses on the crack plane for $\ddot{K}_{I}=0.0042$. Both stress components depend on the distance from the crack tip. Similar distributions have been derived for smaller values of the normalized stress intensity factor. An important feature of $\sigma_{22}$-distribution is the development of a local minimum near the crack tip, followed by a steep increase of the stress level as the tip is approached. This area of large values of hoop stresses on the crack plane is expected to cause hydride fracture and thus crack propagation. In section 5.1 a failure criterion is presented, based on this feature of $\sigma_{22}$-distribution, and the threshold stress intensity factor is predicted.

Figure 6 shows the distribution of hydride volume fraction along the crack line, i.e. along the trajectory line of a material particle, which initially lies on the crack plane and subsequently, after being split by the crack tip, lies on the crack surface. Several distributions for $\bar{K}_{I}$ equal to 0.0042 and 0.0422 as well as for 
$0<N<1$ are presented. In the case of $\bar{K}_{I}=0.0042$, the distribution is derived from the first row of elements, parallel to the crack plane, with thickness equal to $3.64 \cdot 10^{-6}\left(D^{H} / V_{c}\right)$; in the case of $\bar{K}_{I}=0.0422$, the first row of elements has thickness equal to $1.82 \cdot 10^{-4}\left(D^{H} / V_{c}\right)$. Material hardening has a strong effect on the level of hydride volume fraction, behind the crack tip. When the material is purely elastic, i.e. when $N$ equals 1 , the hydrides, which precipitate ahead of the crack tip under the influence of the near-tip field, re-dissolve completely after the crack passes by. However, when the material deforms plastically, re-dissolution of the hydrides behind the crack tip requires additional plastic work, for the reduction of the volume, associated with the phase transformation. This additional work makes re-dissolution more difficult and the hydrides more stable, as compared to the case of purely elastic material behavior. The hydride volume fraction behind the crack tip is the range of 0.60 to 0.75 for a perfectly plastic material and $\bar{K}_{I}<0.0422$.

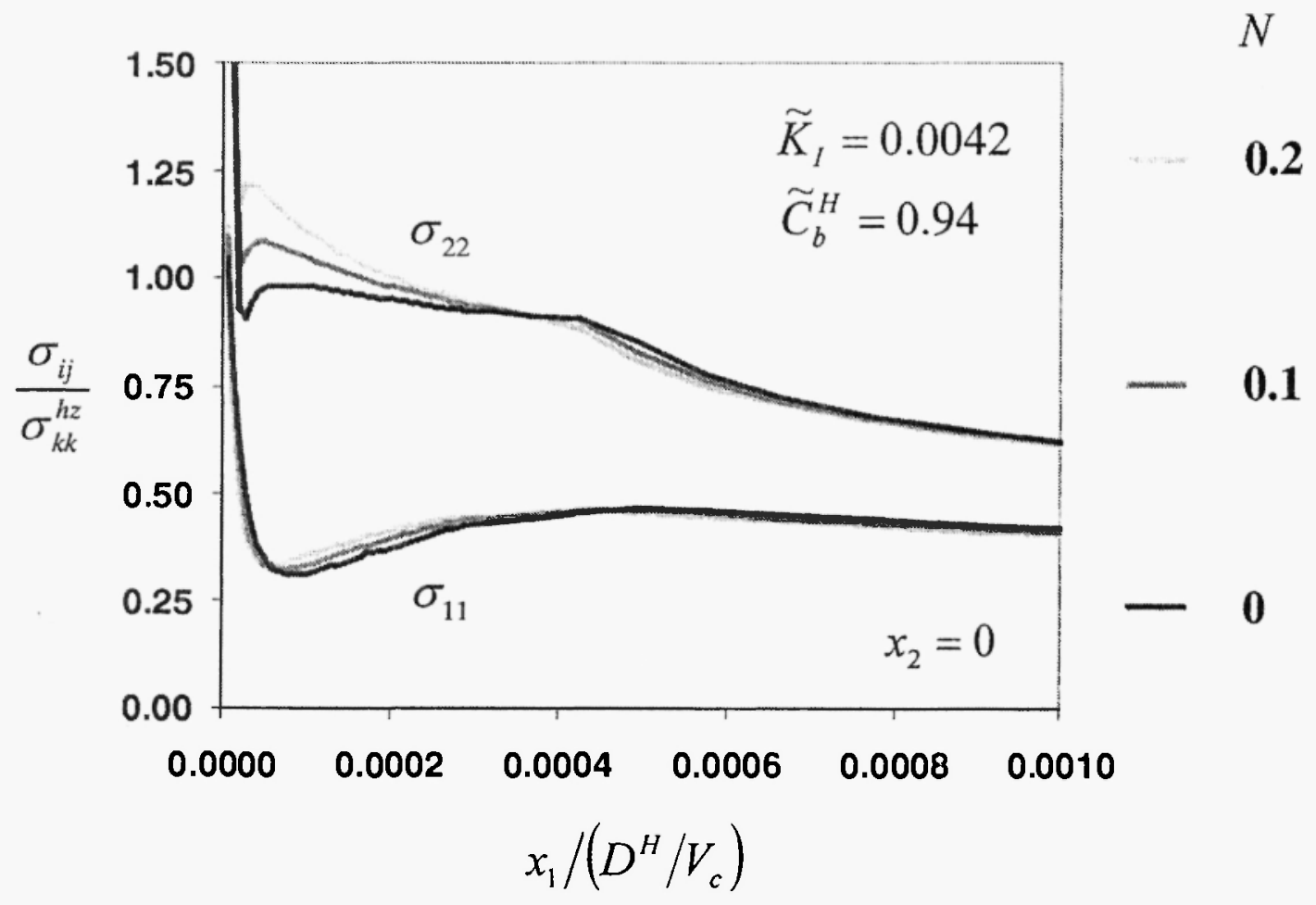

Fig. 5: Normalized distributions of $\sigma_{11}$ and $\sigma_{22}$ ahead of a steadily propagating crack, on the crack plane, in a zirconium alloy. $\mathrm{T}=573 \mathrm{~K} . \bar{K}_{I}=0.0042$ (crack propagation near the threshold). 

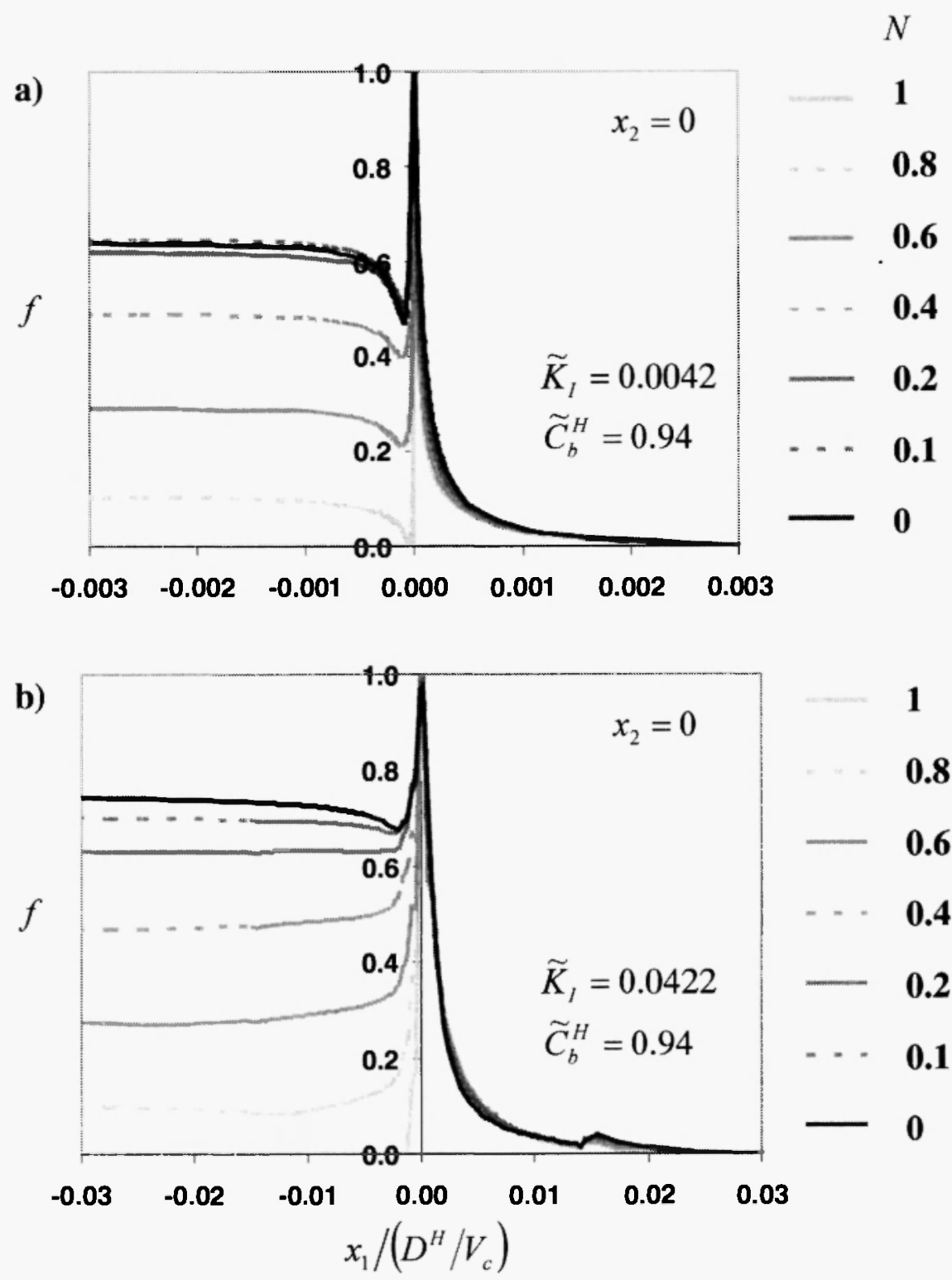

Fig. 6: Normalized distributions of hydride volume fraction on the plane of a crack, propagating steadily in a zirconium alloy. $\mathrm{T}=573 \mathrm{~K}$. (a) $\bar{K}_{I}=0.0042$. (b) $\bar{K}_{I}=0.0422$.

Figure 7 shows the distributions of hydrogen concentration in the solid solution along the trajectory line of a particle on the crack plane. The normalized stress intensity factor takes the same values as those in figures 6. Again the distributions for the whole range of the hardening exponent are presented. Within the zone of hydride precipitation, the concentration of hydrogen in the solid solution is given by the terminal 
solid solubility (relation (8)). Therefore, when $\widetilde{K}_{1}$ tends to zero and thus $\sigma_{k k}$ tends to $\sigma_{k k}^{h z}, C^{H}$ in the hydride precipitation zone tends to a constant value equal to $C^{T S}\left(\sigma_{k k}^{h:}\right)$ (see Figure 7a). Indeed $C^{T S}\left(\sigma_{k k}^{h z}\right)$ $=0.96$. The deviation from $C^{T S}\left(\sigma_{k l}^{h z}\right)$, in the hydride precipitation zone for $\left|x_{1} /\left(D^{H} / V_{c}\right)\right|>6 \cdot 10^{-5}$, at $\ddot{K}_{I}=0.0042$, is less than $0.5 \%$ for the whole range of the hardening exponent. In the case of $K_{I}=0.0013$, not shown in Figure 7, the deviation from $C^{T S}\left(\sigma_{k k}^{h z}\right)$, in the hydride precipitation zone for $\left|x_{1} /\left(D^{H} / V_{c}\right)\right|>1 \cdot 10^{-5}$, is $0.02 \%$ or less, when the material is purely elastic, and $0.1 \%$ or less, when the material is perfectly plastic. Outside the hydride precipitation zone and when $\tilde{K}_{I}$ tends to zero, the concentration of hydrogen follows the distribution, given by relation (16). With the exception of purely elastic material, the effect of hardening exponent on hydrogen concentration in the solid solution is weak to negligible for $K_{I}<0.0422$. This is a direct consequence of the weak to negligible effect of hardening on stress trace, for $\ddot{K}_{I}<0.0422$. In the case of purely elastic material behavior, $N=1$, the distribution of hydrogen concentration, behind the crack tip, is quite different from that of an elastic-plastic material, due to hydride re-dissolution. Note also that, as the normalized stress intensity factor increases, the concentration of hydrogen ahead of the crack tip decreases (see Figure $7 \mathrm{~b}$ ). This is due to the effect of stress trace on hydrogen terminal solid solubility; according to relation (8), an increase of the stress trace leads to a decrease of the terminal solid solubility in the case of a zirconium alloy. As $\bar{K}_{I}$ increases, the stress trace ahead of the crack tip increases (see Figure 4) and, consequently, the hydrogen concentration in the solid solution of the hydride precipitation zone, being equal to the terminal solid solubility, decreases.

\section{DISCUSSION ON FRACTURE CRITERIA}

In section 4.1, the conditions for hydride precipitation ahead of a crack tip were discussed. It was shown that in a perfectly plastic material, hydrides do not precipitate, if the remote hydrogen concentration is below a limit, given by relation (22). Then, relation (22) provides a condition for the suppression of hydride precipitation, ahead of a crack tip in a perfectly plastic material, and consequently for the suppression of subcritical crack growth.

In section 5.1 a fracture criterion is presented for the prediction of the threshold stress intensity factor, when hydrides precipitate ahead of the tip, and therefore, when the remote hydrogen concentration is above the limit, specified by (22).

In section 5.2 another criterion is developed, which allow us the estimation of the crack velocity, during stage II regime.

\subsection{Stress Intensity Factor Threshold of Sub-Critical Crack Growth}

Experiments, performed on zirconium alloys with different chemical composition (Zircaloy-2 and Zircaloy-4) and heat-treatment (stress-relieved, re-crystallized and $\beta$-treated), have shown that there is a 
critical hydrogen concentration at which the material completely loses ductility /18-20/. In several cases the variation of ductility with hydrogen content is rather abrupt, corresponding to a brittle-ductile transition. The critical hydrogen concentration depends on temperature, irradiation and heat treatment. For example, an unirradiated re-crystallized Zircaloy- 2 , at $20^{\circ} \mathrm{C}$, shows an abrupt reduction in ductility, expressed either as elongation or reduction of area at fracture, at about $800 \mathrm{ppm}\left(\cong 5150 \mathrm{~mole} / \mathrm{m}^{3}\right) / 18 /$. The transition of ductility ends at about $1400 \mathrm{ppm}$ and the material is brittle, for larger hydrogen concentration. When the temperature increases, the material is more ductile and the brittle-ductile transition, if exists, moves to larger values of hydrogen concentration. At $300^{\circ} \mathrm{C}$, the brittle-ductile transition of the un-irradiated re-crystallized Zircaloy-2 moves to a hydrogen concentration of about $2000 \mathrm{ppm} / 18 \%$. The material does not show any significant ductility at a hydrogen concentration of $3000 \mathrm{ppm}$ or higher. On the other hand, irradiation causes embrittlement and shifts the critical hydrogen concentration to lower values. At $300^{\circ} \mathrm{C}$, the ductility of the recrystallized Zircaloy-2, mentioned above, is negligible at about $2000 \mathrm{ppm}$, when the material is irradiated. The brittle-ductile transition is attributed to the development of a continuous network of brittle hydrides /1920/. Thus, after the development of the network, fracture occurs by the propagation of a crack along the connected hydrides, without any significant plastic deformation of the solid solution.

Taking into account the above measurements, one concludes that a hydride network, in the re-crystallized Zircaloy- 2 at $300^{\circ} \mathrm{C}$, develops at a hydride volume fraction in the range of 0.1 to 0.2 , if the behavior of both irradiated and un-irradiated material is considered. $f=0.1$ corresponds to the irradiated material.

The critical contents of hydrogen, mentioned above, and therefore the respective values of hydride volume fraction, should only be considered as indicative. Indeed, experiments on Zircaloy-4 with smooth and notched specimens showed a decrease of the critical hydrogen content, at which ductility is lost, by nearly $50 \%$ in the case of notched specimens $/ 21$. Note that, in both specimen types, the hydrides were uniformly distributed. Probably, in the case of notched specimens, larger parts of the fracture surface, among the zones of hydride cleavage, contained dimples, without contributing significantly to the macroscopic strain at fracture. In other words, a hydride-rich area, but not necessarily with a continuous hydride network, may fail with insignificant macroscopic strain, in the presence of stress concentration.

Figure 8 shows the size of the area, $r_{0}(f)$, ahead of the propagating crack on the crack plane, for which the hydride volume fraction is larger than or equal to $f ; r_{0}(f)$ is given as a function of the normalized stress intensity factor. The chosen values of the hydride volume fraction are $0.05,0.1,0.2,0.4$ and 0.6 . Therefore in all cases, the area is very brittle, according to the above experimental measurements. Note that $f=0.05$ could also correspond to complete loss of ductility in the presence of the crack. 


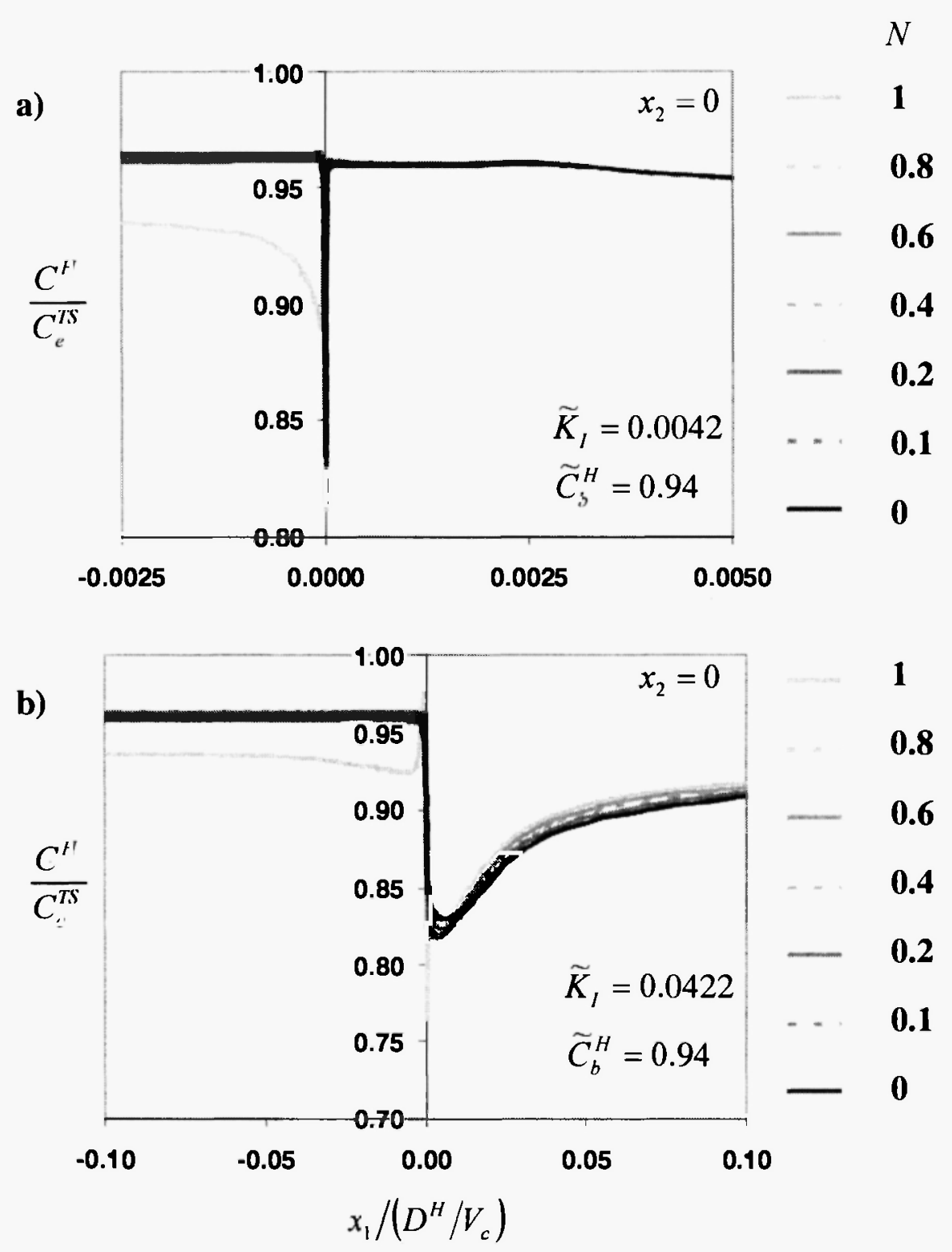

Fig. 7: Normalized distributions of hydrogen concentration in the solid solution on the plane of a crack, propagating steadily in a zirconium alloy. $\mathrm{T}=573 \mathrm{~K}$. (a) $K_{I}=0.0042$. (b) $K_{I}=0.0422$.

When the velocity of the crack tends to zero, consequently when the normalized stress intensity factor tends to zero, the threshold stress intensity factor, $K_{I}^{\text {th }}$, is approached. In this case, Figure 8 a provides information on the size of the brittle, hydride-rich, region ahead of the crack tip, at $K_{\zeta}=K_{I}^{\text {th }}$. For example, if $K_{l}^{t h}=10 \mathrm{MPa} V_{\mathrm{m}}$, then the area with $f \geq 0.10$ has size about equal to $\left.60 \mu \mathrm{m}, \mid \equiv 0.2 \cdot\left(K_{I}^{h} / \sigma_{k k}^{h z}\right)^{2}\right)$. Although very brittle, this region does not fracture, unless the hoop stress of the near-tip field, on the crack 
plane, reaches the strength of the hydrides. According to the discussion in section 4, this may not be the case in the region of stress trace plateau. When the remote hydrogen concentration is equal to $423.72 \mathrm{~mole} / \mathrm{m}^{\text {', }}$
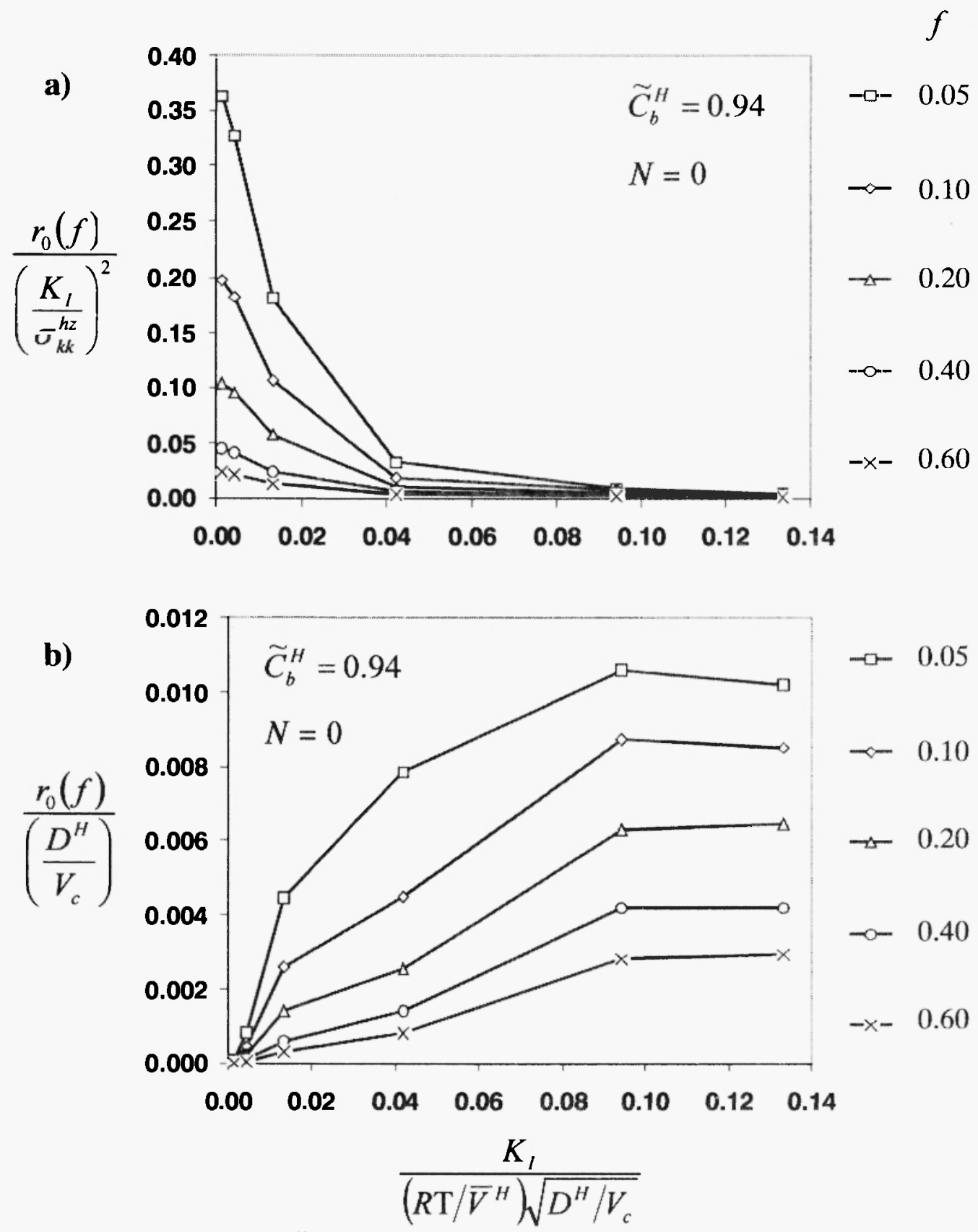

Fig. 8: Variation of the extent, ahead of the crack tip on the crack plane, of the area of hydride precipitation, with hydride volume fraction equal to or larger than $f$, vs. the normalized stress intensity factor. 
(i.e. $65.8 \mathrm{ppm}, \tilde{C}_{b}^{\mu}=0.94$ ), the hoop stress on the crack line, within the stress trace plateau region, has a maximum equal to $555 \mathrm{MPa}$ for a non-hardening material, $\left(K_{I}=0.0013\right)$. It is expected that the hoop stress, in the plateau region, will be even smaller for larger values of the remote hydrogen concentration. On the other hand, the strength of the hydrides is expected to be about $600 \mathrm{MPa}$ or larger (e.g. /22/). However, very close to the crack tip, with the increase of the hydride volume fraction, stresses increase significantly. For $\bar{C}_{b}^{H}=0.94$ and $\bar{K}_{I}=0.0013$, hoop stress increases significantly, when $x_{1} /\left(K_{I} / \sigma_{k k}^{h z}\right)^{2}<A_{\sigma}=10^{-2}$. Note that, in this region, $f>0.87$ and the hoop stress exceeds the above hydride strength values. If an appreciable part of a hydride is within this high stress region, fracture occurs and spreads within the whole hydride-rich region. Let $h$ be the thickness of a hydride. Then, fracture is expected to occur, if a part of the hydride, at least as large as $L_{c}^{\text {th }}=h$, is in the high stress region. Then, the following fracture criterion is considered for estimating the threshold of sub-critical crack propagation:

$$
K_{I}^{t h}=\sigma_{k k}^{h z} \sqrt{\frac{L_{c}^{t h}}{A_{\sigma}}} .
$$

A similar relation is developed if distances are normalized by $\left(K_{I} \bar{V}^{H} / R \mathrm{~T}\right)^{2}$, in which case $\sigma_{k k}^{h i}$ is replaced by $R \mathrm{~T} / \bar{V}^{H}$, in (23). This normalization is more appropriate as $\bar{C}_{b}^{H}$ approaches 1 further. Note that the present analysis is for remote hydrogen concentration below the terminal solid solubility, under no applied stress, i.e. for $\bar{C}_{b}^{H}<1$.

According to experimental measurements of hydride thickness $/ 20 /, h$ is in the range of 0.1 to $1 \mu \mathrm{m}$. Note that these measurements were performed on Zircaloy- 4 and the thickness range varied with the heat treatment. Hydrides $1 \mu \mathrm{m}$-thick or less could also be found in a $\mathrm{Zr}-2.5 \mathrm{Nb}$ alloy (e.g. /23/). Shek et al. /9/ report a thickness of hydride clusters in the range of 1.7 to $3.8 \mu \mathrm{m}$ at $200^{\circ} \mathrm{C}$ as well as in the range of 3 to $4.9 \mu \mathrm{m}$ at $250^{\circ} \mathrm{C}$, in $\mathrm{Zr}-2.5 \mathrm{Nb}$ alloy. Finally, in the case of Zircaloy-2, Efsing /10/ measured hydrides of thickness equal to $4 \mu \mathrm{m}$. If one assumes that $L_{c}^{\text {th }}$ is in the range of 1 to $5 \mu \mathrm{m}$, he calculates, by using relation (23), a stress intensity factor threshold in the range of 5.8 to $13 \mathrm{MPa} V_{\mathrm{m}}$. When $L_{c}^{\text {th }}=4 \mu \mathrm{m}$, according to Efsing $/ 10 /$, then $K_{J}^{\text {th }}=11.6 \mathrm{MPa} \sqrt{\mathrm{m}}_{\mathrm{m}}$.

The above estimated range of the threshold is in good agreement with experimental measurements. Huang and Mills $/ 24 /$ report threshold values between 6.4 and $8.4 \mathrm{MPa} / \mathrm{m}$ for un-irradiated Zircaloy- 2 at $260^{\circ} \mathrm{C}$. No measurement on irradiated material at $260^{\circ} \mathrm{C}$ was performed. However, they measured the threshold for both un-irradiated and irradiated material at $204^{\circ} \mathrm{C}$. For the un-irradiated material, the threshold varied in the range of 5.2 to 7.1 MPa $\sqrt{\mathrm{m}}$. In the case of irradiated Zircaloy-2, one measurement provided a threshold equal to 6 $\mathrm{MPa} V_{\mathrm{m}}$.

Efsing $/ 10 /$ measured the threshold for un-irradiated re-crystallized or stress-relieved Zircaloy- 2 at $300^{\circ} \mathrm{C}$. The threshold increased with the decrease of the yield stress of the material. The stress-relieved alloy, with a yield stress between 490 and $620 \mathrm{MPa}$, showed a threshold in the range of 7.5 to $8.2 \mathrm{MPa} / \mathrm{m}$; note that our 
threshold estimates are for yield stress equal to $580 \mathrm{MPa}$, i.e. within the above range of the un-irradiated stress-relieved Zircaloy-2. Measurements on irradiated Zircaloy-2 at $300^{\circ} \mathrm{C} / 10$ / provided a threshold stress intensity factor equal to $9.9 \mathrm{MPa} \sqrt{\mathrm{m}}$.

\subsection{Stage II Crack Growth Velocity}

It:

Within stage-I growth, the stress distribution changes significantly with the increase of the velocity of the crack and consequently with the increase of the normalized stress intensity factor, $K_{I}$. The near-tip field loses the stress trace plateau as well as the region of steep increase very close to the crack tip. However, according to the discussion in section 4.2 , overall the stress level increases and approaches that of a hydrogen-free material, (see Figure 4). On the other hand, within stage-I, the extent of the hydride-rich region, ahead of the crack tip, decreases with the increase of the velocity, (see Figure 8a).

Within stage-II growth, the velocity of the crack remains nearly constant, under an increase of the applied stress intensity factor, below the fracture toughness of the solid solution. In order to reveal the effect of stageII growth on the hydride-rich region, ahead of the propagating crack, the extent of this region, normalized by $D^{H} / V_{c}$, is presented in Figure $8 \mathrm{~b}$, as a function of the normalized stress intensity factor. Note that the ratio $r_{0}(f) /\left(D^{H} / r_{c}\right)$, for $f=0.05-0.6$, remains nearly constant, when $\bar{K}_{I} \geq \tilde{K}_{I}^{S S}=0.0943$; the deviation from a constant value is $2 \%$ or less for all $f$-values. Based on this feature, one may assume that stage-II growth occurs, when the extent of the hydride-rich area, ahead of the crack tip, remains nearly constant, together with the velocity of the crack, and independent of the applied stress intensity factor. Then $\tilde{K}_{I}^{\text {cs }}$ corresponds to the end of the transition between stage-I and stage-II regime and the beginning of stage-II. If one neglects the variation of the stress intensity factor, during this transition, he derives the following relation for the velocity of the crack, during stage-II growth, $V_{c}^{S S}$ :

$$
{ }_{c}{ }_{c}^{S S}=D^{H}\left(\frac{R \mathrm{~T}}{V^{H}} \frac{\hat{K}_{I}^{S S}}{K_{l}^{t h}}\right)^{2}
$$

Neglecting stage-I/stage-II transition is, for example, a good approximation in the case of the experiments on irradiated Zircaloy -2 at $300^{\circ} \mathrm{C} / 10 \%$. Note that, in $(24), \tilde{K}_{I}^{S S}$ generally depends on temperature, yield stress and hydrogen content. The effects of these parameters on stage-II crack growth velocity is discussed in $/ 14 /$.

Taking into account that, for the material under consideration at $300^{\circ} \mathrm{C}$, the estimated threshold stress intensity factor is in the range of 5.8 to $13 \mathrm{MPa} \mathrm{V}_{\mathrm{m}}$, one may derive, by using (24), that the stage-II crack growth velocity is in the range of $3.3 \cdot 10^{-7}$ to $1.7 \cdot 10^{-6} \mathrm{~m} / \mathrm{s}$. For $K_{l}^{\text {th }}$ equal to $11.6 \mathrm{MPa} V_{\mathrm{m}}$, predicted by assuming a hydride thickness equal to $4 \mu \mathrm{m}$, the calculated velocity is equal to $4.2 \cdot 10^{-7} \mathrm{~m} / \mathrm{s}$.

The estimated velocity range has been derived from calculations under a remote hydrogen concentration of $65.8 \mathrm{ppm}$, which is slightly below hydrogen terminal solid solubility. However, it is in very good 
agreement with experimental measurements, performed for a wide variation of hydrogen content, above the solubility limit. Indeed, the experimental measurements on irradiated Zircaloy- $/ 10 /$, show a stage-II crack growth velocity at $300^{\circ} \mathrm{C}$ equal to $9.5 \cdot 10^{-7} \mathrm{~m} / \mathrm{s}$. Also, the experiments on stress-relieved un-irradiated Zircaloy -2 at $300^{\circ} \mathrm{C} / 10$, which has a yield stress comparable to that considered in the present calculations, showed a stage-II crack growth velocity range of $3 \cdot 10^{-7}$ to $5 \cdot 10^{-6} \mathrm{~m} / \mathrm{s}$.

Most of the velocity measurements of Huang and Mills /24/ on Zircaloy-2 are for un-irradiated material, under lower temperatures. At $288^{\circ} \mathrm{C}$, they measured a stage-II crack growth velocity of about $5 \cdot 10^{-8} \mathrm{~m} / \mathrm{s}$, which is below the estimated range. This is probably because of the low yield stress of the un-irradiated material. Indeed, their testing on irradiated material at $149^{\circ} \mathrm{C}$ and $204^{\circ} \mathrm{C}$ caused a 50 -fold increase on stage-II growth rates.

\section{CONCLUSIONS}

A model for the study of hydride-induced sub-critical crack growth, in metals such as zirconium, titanium, vanadium and niobium, has been developed. The coupling of the operating physical processes of hydrogen diffusion, hydride precipitation and hydride/solid-solution elastic-plastic deformation is taken into account. The model is based on the conditions of constant temperature, plane-strain, small-scale yielding, small-scale hydride precipitation and steady-state crack growth.

A pure metal/hydrogen system has been considered /6-8/. The hydrides are smeared and the development of hydride-rich regions is described by the distribution of the hydride volume fraction. Hydrogen diffusion in the hydrides has been neglected for being significantly slower than the diffusion in the solid solution. Finally, the elastic-plastic properties of the hydride/solid-solution composite were taken to be identical to those of the metal.

The model can be applied to alloys without any changes, if the additional elements do not affect the operating processes. Then, the properties of the alloy with respect to elastic-plastic deformation, hydrogen diffusion and hydride precipitation should be used. This is the case of Zircaloy-2, considered in the present study. It is however possible that, due to alloying and heat treatment, different phases are produced, which have significantly different hydrogen diffusion and hydride precipitation characteristics, like in $\alpha-\beta$ zirconium and titanium alloys. Diffusion in $\beta$-phase is faster and therefore the continuity or not of $\beta$-phase has important implications on hydrogen distribution. In this case the present model should be appropriately modified, in order to take into account the different phase properties as well as information on the microstructure.

It is shown that the distributions of the field quantities, near the tip of a steadily propagating crack, when appropriately normalized, depend on the normalized stress intensity factor, $\bar{K}_{I}=K_{I} \sqrt{V_{c} / D^{H}} /\left(R \mathrm{~T} / V^{H}\right)$. Therefore any variation of the applied stress intensity factor, $K_{I}$, and the crack tip velocity, $V_{c}$, which does not change the value of the normalized stress intensity factor, does not produce any variation in the 
normalized near-tip field distributions. Also the variation of the near-tip field, when the crack propagates from stage-I to stage-II regime, can be studied by varying the normalized stress intensity factor. The near-tip field depends strongly on the ratio of the hydrogen concentration, far from the crack tip, over the terminal solid solubility of hydrogen, under no applied stress, $\tilde{C}_{b}^{I I}=C_{b}^{\prime \prime} / C_{e}^{T S}$.

It is also shown that as the normalized stress intensity factor tends to zero, i.e. as the crack tip velocity tends to zero, a hydrostatic stress plateau develops in the area of hydride precipitation near the crack tip. The plateau is a result of approaching hydrogen chemical equilibrium in the solid solution of the hydride precipitation zone. The plateau hydrostatic stress depends on the normalized remote hydrogen concentration, $\tilde{C}_{b}^{I \prime}$. However, it is independent of the plastic properties of the solid solution. Therefore for a given value of the remote hydrogen concentration, the same hydrostatic stress develops near the crack tip either the material is elastic or elastic-plastic as well as for any level of hardening. The same hydrostatic stress develops also behind the crack tip in the presence of hydrides. The plateau is characteristic of crack propagation near the threshold.

As the normalized stress intensity factor increases, hydrogen distribution deviates from that under chemical equilibrium. The hydrostatic stress also deviates from the thermodynamically required constant value and the stress distributions tend to those of a hydrogen free material.

Based on the thermodynamically required constant hydrostatic stress, which develops near the tip as the crack velocity tends to zero, a critical remote hydrogen concentration has been derived, below which hydride precipitation does not occur ahead of the crack in a perfectly plastic material. Consequently, when the remote hydrogen concentration in a perfectly plastic material is below this critical value, sub-critical crack propagation is suppressed for any level of the applied stress intensity factor.

The characteristics of the near-tip field have also been used for predicting the threshold stress intensity factor as well as the nearly constant crack propagation velocity, during stage-II regime. The predicted range of the threshold stress intensity factor as well as the predicted range of stage-II crack growth velocity, in an irradiated Zircaloy- 2 at $300^{\circ} \mathrm{C}$, is in agreement with experimental measurements.

\section{ACKNOWLEDGMENT}

The present study is part of the project on 'Hydrogen Embrittlement and Fracture in Hydride Forming Metals', financed by the Foundation for Knowledge and Competence Development in Sweden and performed in cooperation with Studsvik Nuclear AB and Barsebäck Kraft AB.

\section{REFERENCES}

1. S. Takano and T. Suzuki, An electron-optical study of $\beta$-hydride and hydrogen embrittlement of 
vanadium. Acta Metallurgica, 22, 265-274 (1974).

2. H.K. Birnbaum, M.L. Grossbeck and M. Amano, Hydride precipitation in $\mathrm{Nb}$ and some properties of NbH. Journal of the Less-Common Metals, 49, 357-370 (1976).

3. D.S. Shih, I.M. Robertson and H.K. Birnbaum, Hydrogen embrittlement of $a$ titanium: in situ TEM studies. Acta Metallurgica, 36, 111-124 (1988).

4. K.G. Denbigh, The Thermodynamics of the Steady State. Methuen, London, 1951.

5. J.C.M. Li, R.A. Oriani and L.S. Darken, The thermodynamics of stressed solids. Zeitschrift für Physikalische Chemie Neue Folge, 49, 271-290 (1966).

6. A.G. Varias and A.R. Massih, Hydride-induced embrittlement and fracture in metals - Effect of stress and temperature distribution. Journal of the Mechanics and Physics of Solids, 50(7), 1469-1510 (2002).

7. A.G. Varias and A.R. Massih, Simulation of hydrogen embrittlement in zirconium alloys under stress and temperature gradients. Journal of Nuclear Materials, 279 (2-3), 273-285 (2000).

8. A.G. Varias, Mathematical model for hydrogen diffusion, energy flow and hydride formation in zirconium under stress. Report BR-04-10-98, Solid Mechanics Research Office, Athens, 1998.

9. G.K. Shek, M.T. Jovanovic, H. Seahra, Y. Ma, D. Li and R.L. Eadie, Hydride morphology and striation formation during delayed hydride cracking in $\mathrm{Zr}-2.5 \% \mathrm{Nb}$. Journal of Nuclear Maicrials, 231, 221-230 (1996).

10. P. Efsing, Delayed hydride cracking in irradiated Zircaloy. Ph.D. Thesis, Royal Institute of Technology, Stockholm, 1998.

11. J.D. Achenbach, P. Burgers and V. Dunayevsky, Near-tip plastic deformations in dynamic fracture problems, in: Nonlinear and Dynamic Fracture, N. Perrone and S. Alturi (Eds.), AMD-35, ASME, New York, 105-124, 1979.

12. M.F. Kanninen and C.H. Popelar, Advanced Fracture Mechanics, Oxford University Press, New York, 1985.

13. A.G. Varias, Steady-state crack growth due to hydride-induced embrittlement - effect of hydride precipitation on near-tip hydrostatic stress. Report PA-01-06-02, Malmö University, Malmö, 2002.

14. A.G. Varias and J.L. Feng, Steady-state crack growth due to hydride-induced embrittlement - effects of temperature, solid solution yield stress and hydride elastic properties. Report PA-01-05-03, Malmö University, Malmö, 2003.

15. A.G. Varias and C.F. Shih, Dynamic steady crack growth in elastic-plastic solids - Propagation of strong discontinuities. Journal of the Mechanics and Physics of Solids, 42, 1817-1848 (1994).

16. A.G. Varias and C.F. Shih, Quasi-static crack advance under a range of constraints - Steady-state fields based on a characteristic length. Journal of the Mechanics and Physics of Solids, 41, 835-861 (1993).

17. W.J. Drugan, J.R. Rice and T.-L. Sham, Asymptotic analysis of growing plane strain tensile cracks in elastic-ideally plastic solids. Journal of the Mechanics and Physics of Solids, 30, 447-473 (1982).

18. S. Arsene and J.B. Bai, Effect of hydriding and irradiation on the mechanical properties of Zircaloy cladding. Report 2, Ecole Central Paris, 1998. 
19. J.B. Bai and D. Francois, Some evidence of a brittle-ductile transition of zirconium hydride between 20 and $350^{\circ} \mathrm{C}$. Journal of Nuclear Materials, 187, 186-189 (1992).

20. J.B. Bai, C. Prioul and D. Francois, Hydride embrittlement in Zircaloy-4: Part I. Influence of microstructure on the hydride embrittlement in Zircaloy- 4 at $20^{\circ} \mathrm{C}$ and $350^{\circ} \mathrm{C}$. Metallurgical and Materials Transactions A, 25A, 1185-1197 (1994).

21. F. Prat, M. Grange, J. Besson and E. Andrieu, Behavior and rupture of hydrided Zircaloy-4 tubes and sheets. Metallurgical and Materials Transactions A, 29A, 1643-1651 (1998).

22. A.G. Varias and A.R. Massih, Temperature and constraint effects on hydride fracture in zirconium alloys. Engineering Fracture Mechanics, 65, 29-54 (2000).

23. D.O. Northwood and U. Kosasih, Hydrides and delayed hydrogen cracking in zirconium and its alloys. International Metals Reviews, 28 (2), 92-121 (1983).

24. F.H. Huang and W.J. Mills, Delayed hydride cracking behavior for Zircaloy-2 tubing. Metallurgical Transactions A, 22A, 2049-2060 (1991). 